\title{
Epidermolysis Bullosa - An anaesthetic challenge
}

\author{
Lokvendra Singh Budania ${ }^{1}$, Eeshwar MV ${ }^{2 *}$,Vamshidhar Chamala Reddy ${ }^{2}$, Yogesh K Gaude ${ }^{1}$, Sai \\ Anand $^{3}$, Anshu Sharma ${ }^{4}$ \\ Associate Professor ${ }^{1}$, Assistant Professor ${ }^{2}$, Junior Resident ${ }^{3}$, Kasturba Medical College, Manipal, India.
}

\begin{abstract}
Epidermolysis bullosa (EB) is a rare genetic disorder characterized by blisters of skin and mucosa as a result of friction or minor trauma. EB can be of three forms: Epidermolysis Simplex (EBS), Junctional Epidermolysis Bullosa (JEB) and Dystrophic (DEB). Management of EB poses a challenge to the anaesthetist. We report a case of a 4-year-old girl who was diagnosed with DEB at birth. She was found to have an oesophageal stricture and was posted for balloon dilatation of the oesophagus. Airway examination revealed mouth opening of 1.5-2 fingers breadth with adequate neck movements. Difficult airway was anticipated. General anaesthesia was administered, and intubation was achieved using Airtraq video laryngoscope. Care was taken not to cause further trauma to the skin and mucosa in the perioperative period.
\end{abstract}

Keywords: Epidermolysis bullosa; difficult airway; airtraq video laryngoscope

\section{Introduction}

Anaesthesia for the paediatric population poses great challenges due to the various anatomical and physiological differences between an adult and a child. A coexisting rare genetic disorder poses a higher risk and poses challenges to the anaesthesiologist. EB is a rare genetic disorder that causes easy bruising of skin and mucosa. ${ }^{1}$ It is associated with multiple complications few of which include, anaemia, recurrent infections, hypermetabolic state, failure to thrive, laryngotracheal scarring, oesophageal strictures, cardiomyopathy each of which requires attention during anaesthesia. ${ }^{2}$ These children come for repeated surgeries and can be subjected to post traumatic stress disorder. ${ }^{3}$ Proper communication with the parents is a must explaining the risk associated with anaesthetic exposure. Therefore, as anaesthesiologists we need to have a holistic approach in managing these children and extend our care beyond the intraoperative period.

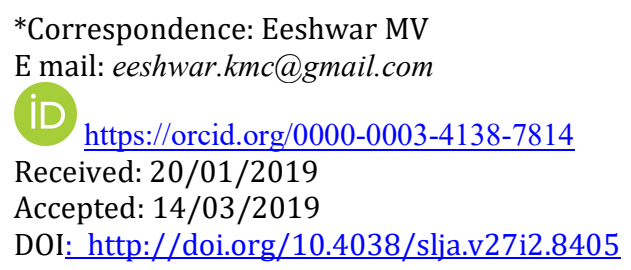

\section{Case report}

A 4-year-old girl presented to our paediatric department with complaints of difficulty in swallowing solids more than liquids for the last 1 year. Her parents gave a history of her being diagnosed with a congenital skin disorder, which was noticed at day 4 of birth. She was diagnosed to have Dystrophic Epidermolysis Bullosa (DEB) and was managed conservatively with regular follow up. A barium swallow revealed a stricture in the oesophagus and she was posted for balloon dilatation. A thorough preoperative anaesthetic workup was done looking for the various complications secondary to DEB. A difficult airway was anticipated in view of restricted mouth opening due to the recurrent skin blisters around her mouth, which had resulted in scarring, but her neck movements were adequate (Figures 1, 2)

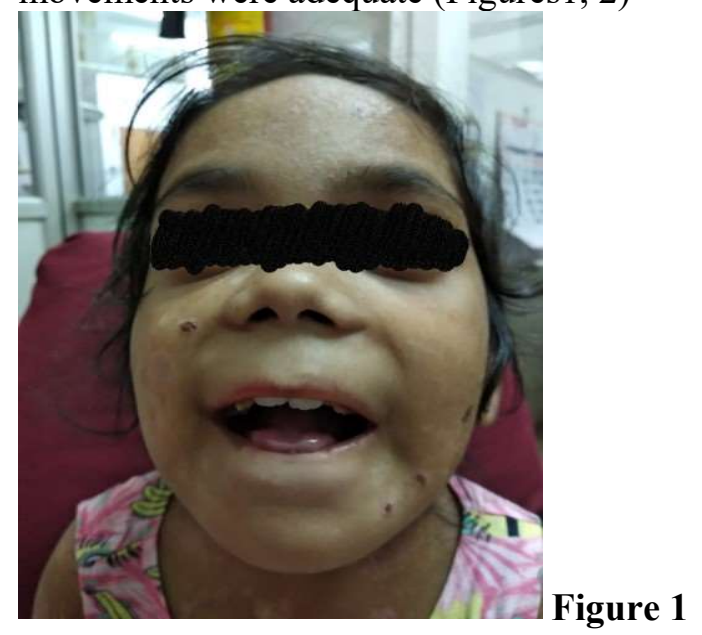




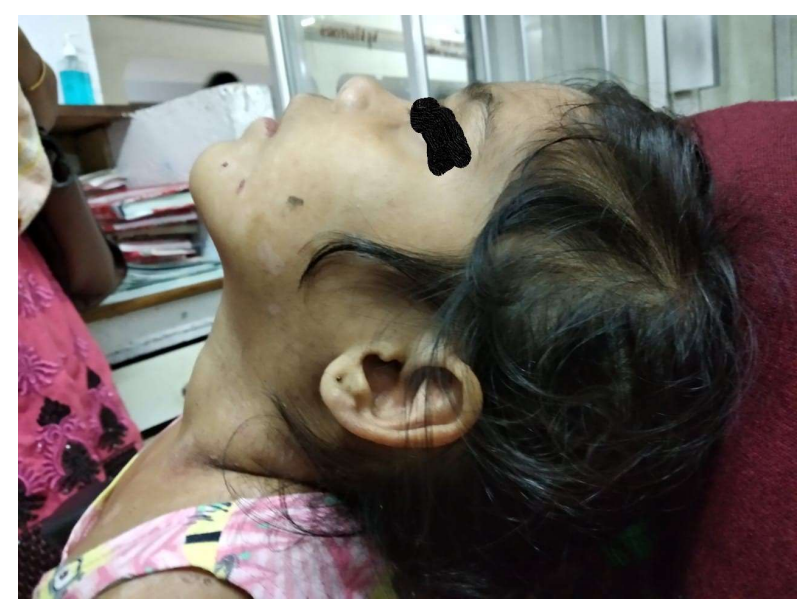

\section{Figure 2}

We had also anticipated a difficult intravenous (IV) access as she had blisters and scarring all over the body. Her general condition was otherwise normal. Plan of anaesthesia was general anaesthesia with endotracheal intubation facilitated by Airtraq video laryngoscope. Her preoperative investigations were normal $\left(\mathrm{Hb} 11 \mathrm{~g} / \mathrm{dL}\right.$, Platelets $250000 / \mathrm{mm}^{3}$, Urea $20 \mathrm{mg} / \mathrm{dL}$, Creatinine $0.4 \mathrm{mg} / \mathrm{dL}$, Serum albumin $3.3 \mathrm{~g} / \mathrm{dL}$ ). The child was kept nil per oral (6hrs for solids and $2 \mathrm{hrs}$ for clear fluids). IV access was obtained over the dorsum of the hand and lactated Ringer's solution was started. Standard monitors (Non-invasive blood pressure, 3 lead ECG, pulse oximeter) were connected and baseline values were recorded. Care was taken to prevent formation of new skin lesions by placing cotton rolls beneath the blood pressure cuff, application of gel to attach ECG electrodes, use of clips for $\mathrm{SpO}_{2}$ monitoring and non-adhesive eye tapes (Figure 3,4).

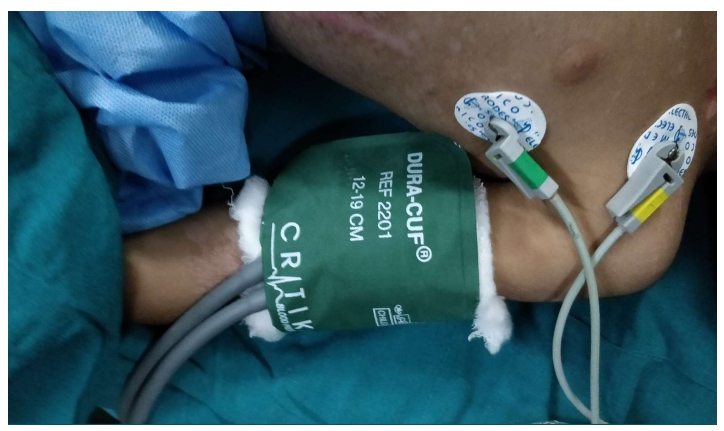

Figure 3

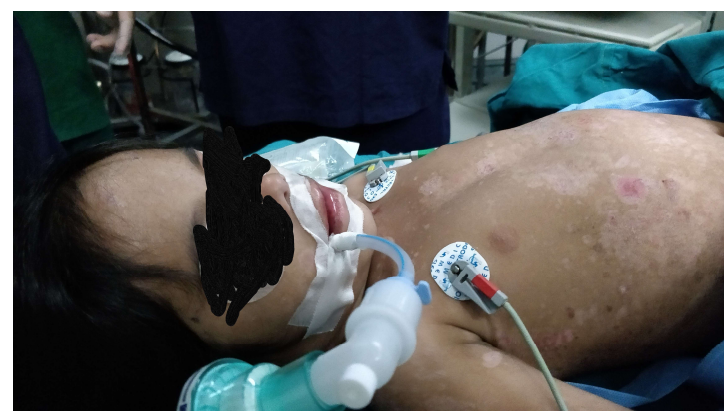

Figure 4

IV propofol $(2-3 \mathrm{mg} / \mathrm{kg})$ with fentanyl $(1-2 \mathrm{mcg} / \mathrm{kg})$ were used for induction. Bag and mask ventilation was confirmed and i.v. atracurium $(0.5 \mathrm{mg} / \mathrm{kg})$ was administered. The child was intubated with a $4.5 \mathrm{~mm}$ uncuffed endotracheal tube using Airtraq video laryngoscopy (percentage of glottis opening score of $100 \%$ ) and anaesthesia was maintained with oxygen, nitrous oxide and isoflurane (Figure 5)

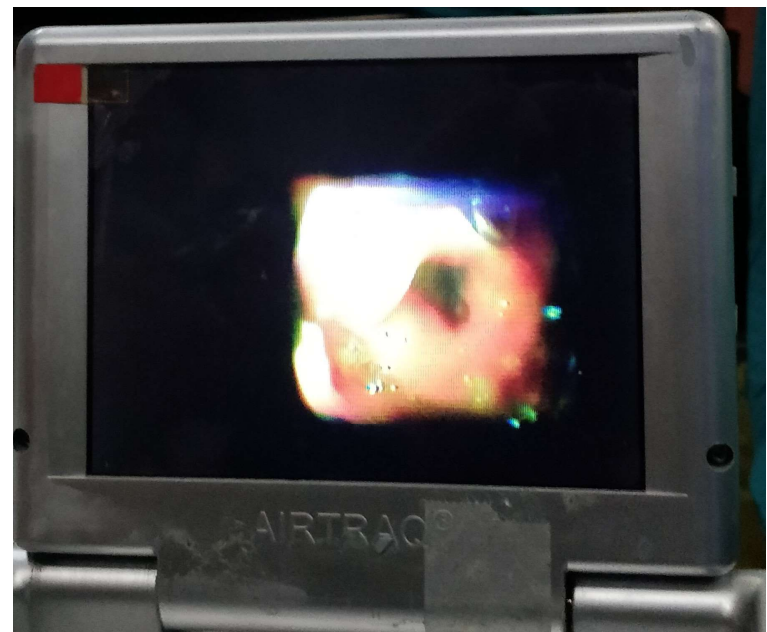

\section{Figure 5}

Severe constriction at the level of cricopharynx was noted and a guide wire was used to negate into the oesophagus after which serial dilatations were performed. IV paracetamol $(20 \mathrm{mg} / \mathrm{kg})$ was administered for analgesia.

\section{Discussion}

EB is mainly classified into 3 types: Simplex, Junctional and Dystrophic epidermolysis bullosa (DEB). The first two variants are the serious forms and result in early mortality whereas the dystrophic variant type progresses into early adulthood. Scarring of tissues and formation of oral and 
mucosal blisters characterize DEB as seen in our case. Some of the common surgical procedures these children come for include contracture release, separation of syndactyly, oesophageal and urethral dilatations, dental and ophthalmic surgeries. Various anaesthetic techniques from simple mask holding, laryngeal mask airway (LMA), endotracheal (ET) intubation or regional anaesthesia have been described. ${ }^{4}$ We wanted to secure the airway in our case as we had anticipated a difficult airway and for oesophageal dilatation the airway needs to be shared with the surgeon.

Preoperative assessment should particularly focus on airway involvement and oral contracture formation. Previous anaesthetic records should be obtained if available. Presence of gastro oesophageal reflux, renal or cardiac involvement should be ruled out. Muscular dystrophy has been reported in a few cases (more so with simplex variant). Few patients might be on steroids, which has anaesthetic implications. Acute epidermolysis is associated with fluid loss and hypoalbuminemia, which needs to be kept in mind. Due to the change in the volume of distribution the dosage of nondepolarizing neuromuscular blockers needs to be titrated. Succinylcholine has been used to facilitate ET intubation. Caution in its use has been advocated due to the risk of hyperkalaemia in children with dystrophies. ${ }^{5}$ Due to the risk of hypoalbuminemia, we had serum albumin levels measured preoperatively.

In our case, we had anticipated a difficult airway and had planned for intubation using a video laryngoscope from the beginning. A fibreoptic bronchoscope and LMA was kept available in the operating room in case of any emergency. As there was no difficulty in ventilating this child we paralysed with a non-depolarizing muscle relaxant. We avoided direct laryngoscopy, which helped avoid trauma to the oral and pharyngeal mucosa and also early use of video laryngoscope aided easy intubation. It is always important to have alternative plans to handle a difficult airway as we had in this scenario. Care was taken to adequately pad the pressure points, use gel for electrodes and nonadhesive tapes to cover the eyes, which prevented formation of new lesions. Thus, it is important to be familiar with such rare disorders, which helps avoiding perioperative morbidity and mortality. ${ }^{6}$

\section{References}

1. Sarkar R, Bansal S, Garg VK. Epidermolysis bullosa: Where do we stand? Indian J of Dermatol, Venerol and Leprol 2011;77; 431-8 https://doi.org/10.4103/0378-6323.82393 PMid:21727690

2. Fine JD, Mellerio JE. Extracutaneous manifestations and complications of inherited epidermolysis bullosa: part I. Epithelial associated tissues. J Am Acad Dermatol. 2009;61(3):367-84 PMID: 19700010 https://doi.org/10.1016/j.jaad.2009.03.052

3. Lansdown R, Atherton D, Dale A, Sproston S, Lloyd J. Practical and Psychological problems for parents of children with epidermolysis bullosa. Child Care Health Dev. 1986; 12(4): 251-56 https://doi.org/10.1111/j.13652214.1986.tb00504.x

4. L. Bowen, M. T. Burtonwood. Anaesthetic management of children with epidermolysis bullosa. BJA Education, 2017;18(2): 41-45 https://doi.org/10.1016/j.bjae.2017.11.005

5. Herod J, Denyer J, Goldman A, Howard R. Epidermolysis bullosa in children: pathophysiology, anesthesia and pain management. Pediatric Anesthesia2002;12(5):388-97 https://doi.org/10.1046/j.1460-9592.2002.00768.x PMid:12060323

6. David Mann, Priscilla J. Garcia, Dean B. Andropoulos. Anesthesia for the patient with a Genetic Syndrome. Gregory's Pediatric Anesthesia. $5^{\text {th }} \quad$ edition 2011;993-1139. https://doi.org/10.1002/9781444345186.ch38 